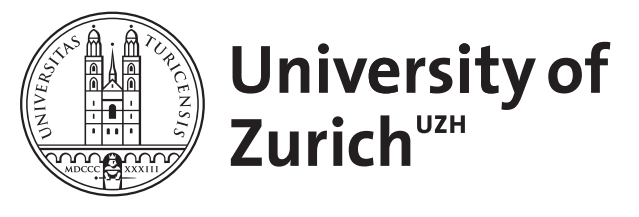

\title{
Chronisches Schmerzsyndrom des Beckens
}

\author{
Walter, M ; Sammer, U ; Kessler, T M
}

\begin{abstract}
Chronic pelvic pain syndrome (CPPS) is defined by the European Association of Urology guidelines as a non-malignant pain perceived in structures related to the pelvis of either women or men for at least 6 months without proven infection or other obvious pathology. It affects the quality of life of millions of people worldwide and has an impact similar to that reported for other chronic diseases, such as diabetes mellitus, Crohn's disease and congestive heart failure. The treatment of CPPS remains a challenge despite several established first line therapies because many patients are therapy refractory. Unconventional treatments, such as neurostimulation, neuromodulation and acupuncture may be highly successful for treating CPPS and have a favorable adverse event profile. Thus, these promising therapeutic alternatives should be considered more often in daily clinical practice.
\end{abstract}

DOI: https://doi.org/10.1007/s00120-012-3037-9

Other titles: Chronic pelvic pain syndrome: neurostimulation, neuromodulation and acupuncture

Posted at the Zurich Open Repository and Archive, University of Zurich

ZORA URL: https://doi.org/10.5167/uzh-72437

Journal Article

Published Version

Originally published at:

Walter, M; Sammer, U; Kessler, T M (2012). Chronisches Schmerzsyndrom des Beckens. Der Urologe. Ausg. A, 51(12):1683-1691.

DOI: https://doi.org/10.1007/s00120-012-3037-9 


\section{Leitthema}

Urologe 2012 · 51:1683-1691

DOI 10.1007/s00120-012-3037-9

Online publiziert: 7. Dezember 2012

c) Springer-Verlag Berlin Heidelberg 2012
M. Walter • U. Sammer - T.M. Kessler

Neuro-Urologie, Zentrum für Paraplegie, Uniklinik Balgrist, Universität Zürich, Zürich

\section{Chronisches Schmerz- syndrom des Beckens}

\section{Neurostimulation, Neuromodulation und Akupunktur}

Das chronische Schmerzsyndrom des Beckens (",chronic pelvic pain syndrome", CPPS) ist gemäß den Richtlinien der „European Association of Urology" (EAU) als ein nicht-maligner Schmerz definiert, welcher in Bezug auf die Strukturen des weiblichen oder männlichen Beckens über einen Zeitraum von mindestens 6 Monaten wahrgenommen wird, ohne dass eine Infektion oder offensichtliche anderweitige Pathologie vorliegt [1, 2].

Der Begriff des CPPS wurde erstmals 1995 vom „National Institute of Diabetes and Digestive and Kidney Diseases“ (NIDDK), einem Teilbereich des „National Institute of Health“ (NIH), in der Klassifikation der Prostatitis etabliert [3].

Im Jahr 2007 wurde durch das NIDDK im Rahmen eines Forschungsprojekts, welches zum besseren Verständnis des CPPS in der Urologie führen sollte, der Begriff des „urological chronic pelvic pain syndrome“ (UCPPS) eingeführt, welcher sich speziell auf die Schmerzsymptomatik ausgehend von der Harnblase und der Prostata bezieht [4]. Doch was genau verstehen wir unter chronischem und/oder urogenitalem Schmerz? Der Schmerz ist eine unangenehme sensorische, emotionale Erfahrung, die mit einer vorliegenden oder potentiellen Gewebeschädigung oder -verletzung einhergeht [5]. Es gibt keine einheitliche internationale Definition, nach welcher Zeit ein Schmerz als „chronisch“ gilt. Einerseits gehen die
"International Association for the Study of Pain“ [6] und „The British Pain Society“ [7] von mindestens 3 Monaten, andererseits die EAU $[1,2]$, die „American Urological Association" (AUA [8]) und das „American College of Obstetricians and Gynecologists" [9] von mindestens $6 \mathrm{Mo}$ naten aus.

Das CPPS wurde initial als ein harnblasen- oder prostataassoziierter Schmerz verstanden. Jahrelange Forschung, die sich mit der Hypothese einer infektiösen Ätiologie beschäftigte, führte jedoch zu keinem nachweislichen Ergebnis. Heutzutage werden andere Auffassungen ver- treten [10]. So scheinen multiple Faktoren in die Pathogenese des CPPS involviert zu sein wie z. B. chemische Reizmittel, endokrine Faktoren, Veränderungen innerhalb der Beckenbodenmuskulatur sowie immunologische und neurologische Einflüsse. Entsprechend dem breiten Spektrum von vermutlichen Auslösern wurde eine große Zahl von Therapien propagiert: $\mathrm{z}$. B. Analgetika, Antibiotika, Alphablocker, 5aReduktasehemmer, intravesikale Instillationen, Elektrotherapien, transurethrale Interventionen und weit eingreifende Operationen wie die Zystektomie - aber nur mit limitierter Wirksamkeit. Weitaus

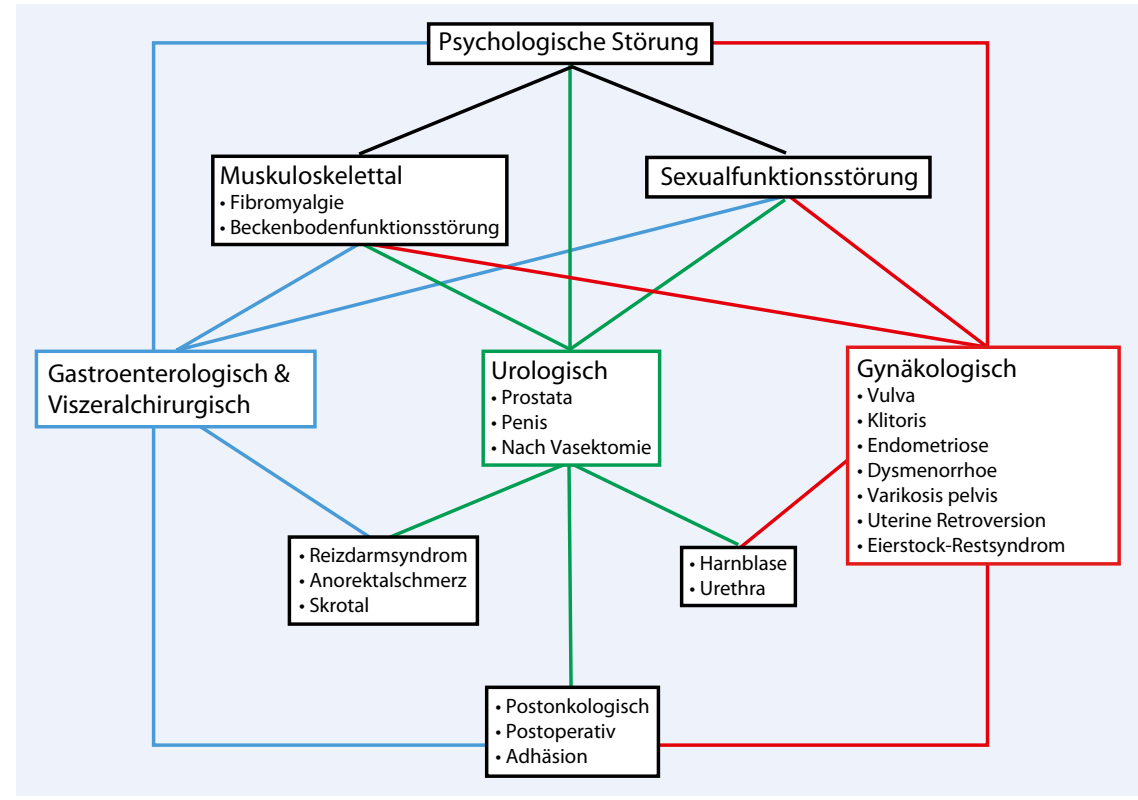

Abb. $1 \Delta$ CPPS-Netzwerk 
erfolgreicher scheinen Kombinationstherapien zu sein, wie z. B. die Kombination eines Antibiotikums und eines Alphablockers als Therapie der ersten Wahl bei Männern mit CPPS [11].

Nicht allein wegen der anatomischen Gegebenheiten, sondern auch wegen den Überschneidungen [12] der klinischen Präsentation urologischer und nicht-urologischer Erkrankungen, ist das CPPS ein inter- bzw. multidisziplinäres Problem, das in den letzten Dekaden an Bedeutung im klinischen Alltag und Interesse in der Forschung gewonnen hat (• Abb. 1). Wir Ärzte sind gefordert, nach fokussierter Anamnese und Diagnostik die Anzahl möglicher Ursachen zu limitieren und eine zielgerichtete Therapie einzuleiten.

Das CPPS beeinträchtigt die Lebensqualität von Millionen von Menschen weltweit und hat einen ähnlich großen Einfluss wie andere chronische Krankheiten (z. B. Diabetes mellitus, Morbus Crohn oder kongestive Herzinsuffizienz $[13,14])$. Inzidenz und Prävalenz variieren von Land zu Land $[5,15]$. So liegt z. B. die Prävalenz in Österreich bei Frauen um 6\% und bei Männern um 3\% [16].

Trotz mehrerer etablierter Erstlinientherapien bleibt die Behandlung des CPPS eine große Herausforderung, sind doch 20-65\% der Patienten therapierefraktär $[17,18]$. So ist es unser Ziel, unkonventionelle Behandlungsverfahren des CPPS wie Neurostimulation, Neuromodulation und Akupunktur näher zu beleuchten.

\section{Neurostimulation und Neuromodulation}

Neurostimulation und Neuromodulation werden oft synonym verwendet, doch es gibt wichtige Unterschiede: Bei der Neurostimulation wird die Funktion eines Neurons direkt beeinflusst, d. h. es kommt zum direkten Effekt am Zielorgan [z. B. Kontraktion des Detrusors durch Stimulation der Vorderwurzeln (Brindley-Vorderwurzelstimulator)]. Bei der Neuromodulation wird die Funktion nachgeschalteter Neurone durch Stimulation eines Neurons beeinflusst, d. h. moduliert. Es kommt zum indirekten Effekt am Zielorgan.

Für unsere Literaturrecherche verwendeten wir Pubmed (Search-Kriterien:

Urologe 2012·51:1683-1691 DOI 10.1007/s00120-012-3037-9

(c) Springer-Verlag Berlin Heidelberg 2012

\section{Walter $\cdot$ U. Sammer $\cdot$ T.M. Kessler \\ Chronisches Schmerzsyndrom des Beckens. Neurostimulation, Neuromodulation und Akupunktur}

\section{Zusammenfassung}

Das chronische Schmerzsyndrom des Beckens ("chronic pelvic pain syndrome", CPPS) ist gemäß den Richtlinien der "European Association of Urology" charakterisiert als ein nicht-maligner Schmerz, der in Bezug auf die Strukturen des weiblichen oder männlichen Beckens über einen Zeitraum von mindestens 6 Monaten wahrgenommen wird, ohne dass eine Infektion oder offensichtliche anderweitige Pathologie vorliegt. Das CPPS beeinträchtigt die Lebensqualität von Millionen von Menschen weltweit und hat einen ähnlich großen Einfluss wie andere chronische Krankheiten, z. B. Diabetes mellitus, Morbus Crohn oder kongestive Herzinsuffizienz. Trotz mehrerer etablierter Erstlinientherapien stellt die Behandlung des CPPS eine große Herausforderung dar, da viele Patienten therapierefraktär bleiben. Unkonventionelle Behandlungsmethoden wie Neurostimulation, Neuromodulation und Akupunktur können beim CPPS eine ausgezeichnete Wirkung zeigen und haben ein vorteilhaftes Nebenwirkungsprofil. So sollten diese vielversprechenden Therapieverfahren im klinischen Alltag vermehrt eingesetzt werden.

\section{Schlüsselwörter}

Nicht-maligner Schmerz • Becken ·

Neurostimulation - Neuromodulation . Akupunktur

\section{Chronic pelvic pain syndrome. Neurostimulation, neuromodulation and acupuncture}

\section{Abstract}

Chronic pelvic pain syndrome (CPPS) is defined by the European Association of Urology guidelines as a non-malignant pain perceived in structures related to the pelvis of either women or men for at least 6 months without proven infection or other obvious pathology. It affects the quality of life of millions of people worldwide and has an impact similar to that reported for other chronic diseases, such as diabetes mellitus, Crohn's disease and congestive heart failure. The treatment of CPPS remains a challenge despite several established first line therapies be- cause many patients are therapy refractory. Unconventional treatments, such as neurostimulation, neuromodulation and acupuncture may be highly successful for treating CPPS and have a favorable adverse event profile. Thus, these promising therapeutic alternatives should be considered more often in daily clinical practice.

\section{Keywords}

Non-malignant pain - Pelvis .

Neurostimulation · Neuromodulation .

Acupuncture „chronic pelvic pain syndrome“, „neurostimulation“, „neuromodulation“, „acupuncture“) und schlossen nur Originaloder Übersichtsarbeiten in deutscher oder englischer Sprache ein.

\section{Transkranielle Stimulation durch Strom oder Magnetfeld}

Die transkranielle Stimulation durch Strom („transcranial direct current stimulation“, tDCS) zur Behandlung des CPPS wurde bisher nur in einer Studie untersucht. Im Rahmen einer prospektiven, randomisierten, doppelt verblindeten Crossover-Studie wurden 7 therapierefraktäre CPPS-Patienten mit einem
Durchschnittsalter von 38 Jahren eingeschlossen [19]. Die aktive Therapie wurde über einen Zeitraum von 2 Tagen für jeweils 20 min mittels direkter Stromapplikation von $1 \mathrm{~mA}$ durchgeführt. In der Sham-Gruppe wurde die Stimulation bereits nach $30 \mathrm{~s}$ wieder beendet. In der aktiven Therapiegruppe zeigte sich im Vergleich zur Sham-Gruppe auf einer visuellen Analogskala (VAS) eine signifikante Reduktion der Becken- und Gesamtschmerzsymptomatik. Nebenwirkungen wie Kopf- und Nackenschmerzen, Brennen und Schmerzen der Kopfhaut traten in beiden Gruppen gleichhäufig auf. Es kam zu keinem nebenwirkungsbedingten Studienabbruch. 
Hier steht eine Anzeige.

第 Springer 
Tab. 1 Originalstudien sakrale Neuromodulation bei Patienten mit CPPS

\begin{tabular}{|c|c|c|c|c|c|c|c|c|}
\hline Autoren & Jahr & Typ & $\begin{array}{l}\text { Patienten mit } \\
\text { CPPS [n (\%)] }\end{array}$ & $\begin{array}{l}\text { Follow-up } \\
\text { (Monate) }\end{array}$ & $\begin{array}{l}\text { Kontroll- } \\
\text { gruppe }\end{array}$ & $\begin{array}{l}\text { Erfolgreiche Test- } \\
\text { phase [n (\%)] }\end{array}$ & $\begin{array}{l}\text { Erfolgreiche perma- } \\
\text { nente SNM [n (\%)] }\end{array}$ & $\begin{array}{l}\text { Komplika- } \\
\text { tionsrate (\%) }\end{array}$ \\
\hline Cardarelli et al. [32] & 2012 & RS & $12 / 157(8)$ & 11 & - & k.A. & k.A. & k.A. \\
\hline Martellucci et al. [40] & 2012 & PS & $27(100)$ & 37 & - & $16 / 16(100)$ & $16 / 16(100)$ & 0 \\
\hline Otto et al. [41] & 2012 & PS & $7 / 47(15)$ & 11 & - & $4 / 7(57)$ & $4 / 4(100)$ & k.A. \\
\hline Powell und Kreder [46] & 2010 & RS & k.A. & 60 & - & $39 / 39(100)$ & k.A. & k.A. \\
\hline Kessler et al. [38] & 2008 & PS & $9 / 44(20)$ & 31 & - & k.A. & k.A. & k.A. \\
\hline Pham et al. [45] & 2008 & RS & $2 / 124(2)$ & k.A. & - & $1 / 2(50)$ & - & - \\
\hline Zabihi et al. [49] & 2008 & RS & k.A. & 24 & - & k.A. & k.A. & k.A. \\
\hline Kessler et al. [37] & 2007 & PS & $17 / 209(8)$ & 10 & - & $7 / 17(41)$ & $5 / 7(71)$ & k.A. \\
\hline Peters et al. [43] & 2007 & PS & $22(100)$ & 15 & Pudendal & $17 / 17(100)$ & $4 / 4(100)$ & $2^{a}$ \\
\hline Elhilali et al. [34] & 2005 & RS & $2 / 52(5)$ & 78 & - & k.A. & $1 / 2(50)$ & k.A. \\
\hline Lavano et al. [50] & 2005 & PS & $7(100)$ & 8 & - & $5 / 5(100)$ & $5 / 5(100)$ & $3^{a}$ \\
\hline Peters et al. [42] & 2005 & PS & k.A. & k.A. & Pudendal & k.A. & $5 / 5(100)$ & $3^{\mathrm{a}}$ \\
\hline Everaert et al. [36] & 2004 & PS & k.A. & 24 & - & k.A. & k.A. & k.A. \\
\hline Peters u. Konstandt [44] & 2004 & RS & $21(100)$ & 15 & - & k.A. & $20 / 21(95)$ & k.A. \\
\hline Comiter [33] & 2003 & PS & $25(100)$ & 14 & - & $17 / 17(100)$ & $16 / 17(94)$ & 0 \\
\hline Scheepens et al. [47] & 2002 & PS & k.A. & k.A. & $\begin{array}{l}\text { Unilateral } \\
\text { vs. bilateral }\end{array}$ & k.A. & k.A. & k.A. \\
\hline Aboseif et al. [31] & 2002 & RS & k.A. & 24 & - & k.A. & $41 / 41(100)$ & k.A. \\
\hline Everaert et al. [35] & 2001 & RS & $26(100)$ & k.A. & - & $16 / 16(100)$ & $8 / 11(73)$ & $1 / 11(9 \%)$ \\
\hline Siegel et al. [48] & 2001 & PS & $10(100)$ & 19 & - & k.A. & 9/10 (90) & $27^{a}$ \\
\hline Maher et al. [39] & 2000 & PS & $15(100)$ & k.A. & - & $13 / 13(100)$ & k.A. & 0 \\
\hline
\end{tabular}

Bisher liegt keine Studie zur Evaluation der transkraniellen Stimulation durch ein Magnetfeld („transcranial magnetic stimulation“, TMS) beim CPPS vor.

\section{Tiefe Hirnstimulation}

Bei der tiefen Hirnstimulation („deep brain stimulation“, DBS), die bereits seit den 70er Jahren [20] des letzten Jahrhunderts eingesetzt wird, werden eine oder mehrere Elektroden in eine bestimmte Hirnregion implantiert, so dass dauerhaft elektrische Impulse abgegeben werden können, die je nach dem stimulierend oder deaktivierend wirken. Diese Methode wird v. a. bei der Behandlung verschiedener therapierefraktärer Bewegungsstörungen wie bei Morbus Parkinson, essentiellem Tremor und Dystonie eingesetzt. Mehrere Studien zeigten, dass die DBS auch die Blasenfunktion beeinflussen kann [21, 22, 23]. Ferner konnten mit der DBS, wie bereits vor vielen Jahren von Hosobuchi [24] beschrieben, bei Patienten mit therapierefraktären Schmerzen, u. a. auch im Beckenbereich, Behandlungserfolge erzielt werden. Aller- dings bleibt unklar, ob mittels DBS speziell auch das CPPS angegangen werden kann. Bis dato gibt es keine Studie bei dieser Patientenpopulation.

\section{Transkutane elektrische Nervenstimulation}

Sikiru et al. [25] untersuchten in einer prospektiven, randomisierten, doppelt verblindeten, placebokontrollierten Studie die Wirksamkeit der transkutanen elektrischen Nervenstimulation („transcutaneous electrical nerve stimulation“, TENS) zur Behandlung des CPPS. Es wurden 24 Patienten im Alter von 24-50 Jahren eingeschlossen und in drei Gruppen zu je 8 Patienten randomisiert: A) Antibiotikum (Ofloxacin $300 \mathrm{mg} / \mathrm{Tag}$ ) und TENS; B) Antibiotikum und Analgetikum (Ibuprofen $400 \mathrm{mg} / \mathrm{Tag}$ ) und C) Antibiotikum und Placebo. TENS wurde jeweils 20 min lang, 5-mal/Woche über den Zeitraum von 4 konsekutiven Wochen appliziert. Es konnte eine signifikante Verbesserung $(\mathrm{p}<0,05)$ der Beschwerden in der TENS-Gruppe im direkten Vergleich zur
Analgetikum- und Placebo-Gruppe nachgewiesen werden.

\section{Perkutane tibiale Nervenstimulation}

Kim et al. [26] zeigten anhand von $15 \mathrm{~Pa}$ tienten mit einem Durchschnittsalter von 60 Jahren nach einer 12-wöchigen Therapie mit der perkutanen tibialen Nervenstimulation ("percutaneous tibial nerve stimulation", PTNS) einmal/Woche jeweils 30 min eine signifikante $(\mathrm{p}<0,05)$ Reduktion der Schmerzen, wobei 9/15 eine Verbesserung von $>50 \%$ und $3 / 15$ zwischen 25 und $50 \%$ beschrieben. In einer vom Design ähnlichen Studie von Balken et al. [27] mit 33 Patienten mit einem Durchschnittsalter von 52 Jahren waren die Resultate negativer mit einer Verbesserung von knapp 40\% (7/33 Verbesserung von $>50 \%$ und $6 / 33$ zwischen 25 und 50\%). Pannek u. Nehiba [28] untersuchten ein gemischtes Kollektiv von 8 Patienten, wobei 3 an einem CPPS litten. Bei allen $3 \mathrm{~Pa}$ tienten nahmen die Schmerzen ab, bei keinem konnte jedoch die Schmerzmedikation umfangreich reduziert oder abge- 
setzt werden. Ferner zeigten von Balken et al. [29], dass bei CPPS-Patienten durch PTNS auch eine Verbesserung der Sexualfunktion erreicht werden kann (subjektiv bei $9 / 23$, objektiv bei $5 / 23$ ).

\section{Sakrale Neuromodulation}

Die sakrale Neuromodulation („sacral neuromodulation“, SNM) stellt ein mittlerweile gut etabliertes Therapieverfahren für therapierefraktäre Blasen- und Darmfunktionsstörungen dar [30]. Bei der SNM wird eine Elektrodenimplantation ins Sakralforamen S3 oder S4 durchgeführt und in einer Testphase über Tage bis Wochen geprüft, ob sie dem Patienten einen relevanten Nutzen bringt. Wenn sich eine positive Testphase zeigt, wird der Neuromodulator gluteal (oder seltener in die Bauchdecke) implantiert.

Im Vergleich zu Blasen- und Darmfunktionsstörungen wurde der Effekt der SNM auf das CPPS nur selten untersucht. Wir haben 20 Originalarbeiten [31, 32, 33, $34,35,36,37,38,39,40,41,42,43,44,45$, $46,47,48,49,50]$, davon 12 prospektive $[33,36,37,38,39,40,41,42,43,47,48$, $50]$ und 8 retrospektive [31, 32, 34, 35, 44, $45,46,49]$ Studien analysiert, wobei nur 3 eine Kontrollgruppe aufwiesen ([42, 47, 51], - Tab. 1). Insgesamt wurden in diese 20 Studien 1242 Patienten eingeschlossen, davon 202 (16\%) mit CPPS. Wichtig ist zu berücksichtigen, dass das Studiendesign dieser Arbeiten uneinheitlich ist und viele Angaben fehlen. Die wichtigsten Ergebnisse sind im Folgenden zusammengefasst:

- mittleres Alter 52 (42-67) Jahre

- Geschlecht: 620 Frauen, 162 Männer, bei 460 Patienten keine differenzierte Angabe
- Testphase:

- Dauer: durchschnittlich 15 (7-47) Tage

- Erfolgsrate: durchschnittlich 58\% (37-87\%), für CPPS allein durchschnittlich 91\% (41-100\%)

- Komplikationsrate: durchschnittlich 5\% (0-9\%), für CPPS allein keine Angabe

- Permanente SNM:

- Implantationsstelle: meist S3, selten S2 oder S4

- Neuromodulationsart:meist unilateral, seltener bilateral

- Follow-up: durchschnittlich 27 (878) Monate

- Erfolgsrate: durchschnittlich $81 \%$ (46-100\%), für CPPS allein durchschnittlich 91\% (50-100\%)

- Komplikationsrate:durchschnittlich 15\% (9-50\%), für CPPS allein keine Angabe

Mit einer Durchschnittserfolgsrate von 91\% scheint die SNM eine ausgezeichnete Therapieoption bei Patienten mit CPPS darzustellen. Allerdings ist zu berücksichtigen, dass die Patientenzahlen relativ klein sind und es insbesondere nur wenige Langzeitresultate gibt.

\section{Pudendale Nervenstimulation}

Die pudendale Nervenstimulation („pudendal nerve stimulation“, PNS) stellt eine vielversprechende therapeutische Alternative zur SNM dar bzw. kann nach erfolgloser SNM erwogen werden [43]. Peters et al. [42, 43] verglichen im Rahmen von 2 prospektiven, randomisierten, einfach verblindeten Crossover-Studien PNS und SNM bei Patienten mit Blasenfunktionsstörungen und CPPS. In der ersten
Studie [42] zeigte sich bei $80 \%$ (24/30) der 30 Patienten mit einem Durchschnittsalter von 51 Jahren eine erfolgreiche Testphase, so dass der Neuromodulator implantiert wurde. Von diesen 24 Patienten entschieden sich mehr für eine PNS als für eine SNM ( $n=19$ vs. 5), da sich die Beschwerden häufiger durch die PNS als durch die SNM (63 vs. $46 \%, \mathrm{p}=0,02$ ) besserten. Dies traf auch für die Subgruppe der CPPS-Patienten $\mathrm{zu}(\mathrm{p}=0,024)$. In der zweiten Studie [43] wurden 22 Patienten mit einem Durchschnittsalter von 48 Jahren untersucht. Die Testphase war erfolgreich in $77 \%$ (17/22). Wiederum war die PNS im Vergleich zur SNM (59 vs. $44 \%$, $\mathrm{p}=0.05)$ effektiver, so dass sich mehr für eine PNS als für eine SNM ( $\mathrm{n}=13$ vs. 4 ) entschieden.

In einer retrospektiven Analyse von 84 CPPS-Patienten mit einem Durchschnittsalter von 52 Jahren zeigte sich eine erfolgreiche Testphase der PNS in insgesamt 71\% (60/84) und bei jenen mit vorherig unwirksamer SNM sogar in 93\% (41/44).

Nach der Testphase entschieden sich $92 \%$ (55/60) für eine PNS und die übrigen für eine SNM. Im Follow-up nach $12 \mathrm{Mo-}$ naten waren bis auf die Urininkontinenz $(\mathrm{p}=0,58)$ und den Schmerz $(\mathrm{p}=0,13)$ die Miktionsverhältnisse signifikant (mindestens $\mathrm{p}<0,002)$ verbessert [52].

\section{Akupunktur}

Die Akupunktur [abgeleitet vom Lateinischen „acus“ (Nadel) und „punctio“ (Stechen)] ist eine traditionelle chinesische Behandlungsmethode, die vor mehr als 2000 Jahren entwickelt wurde [53]. Unter der Annahme, dass sich in unserem Körper die Lebensenergie (气, qi) auf de-

\section{Hier steht eine Anzeige.}




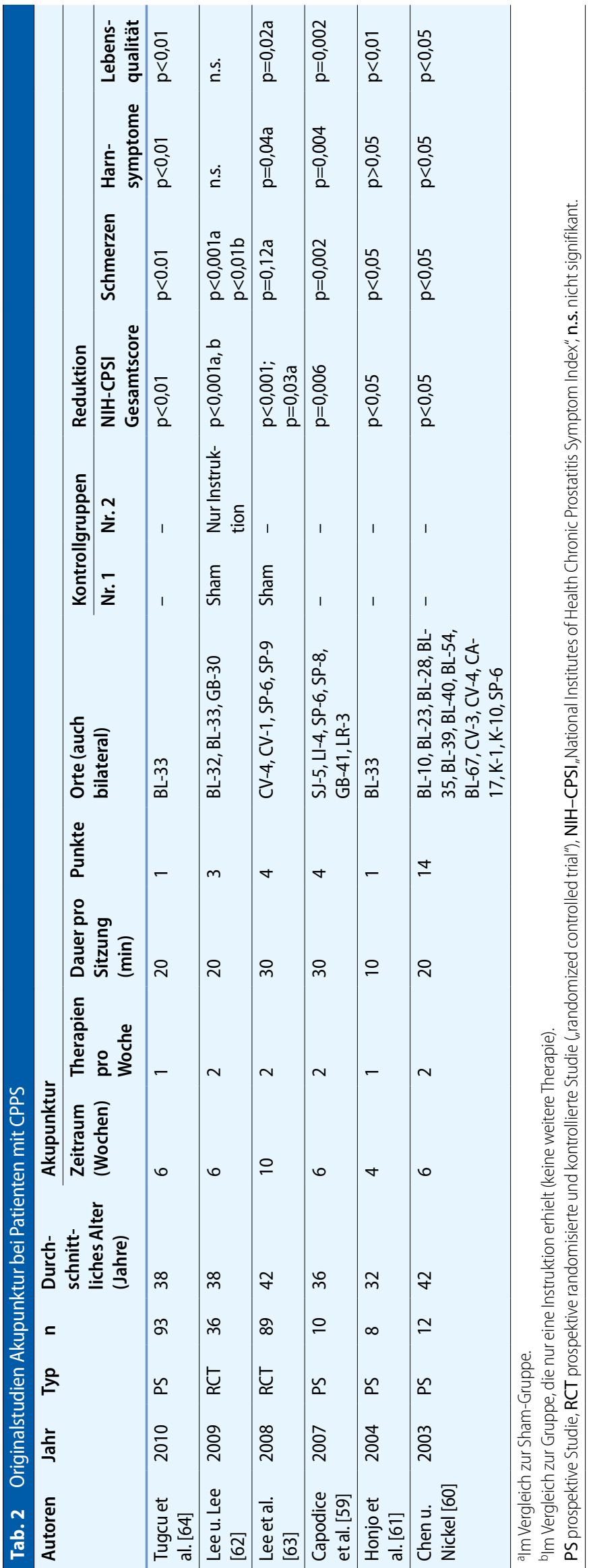

finierten Längebahnen (Meridianen 经络, jing luò) bewegt und einen regulierenden Einfluss auf spezifische Körperfunktionen ausübt, kann ein im Rahmen von Erkrankungen vorliegender gestörter Energiefluss durch gezielte Insertionen von speziellen Nadeln in ausgewählte(n) Akupunkturpunkt(e) (穴位, xué wèi) behoben werden [54]. Zur Insertion werden feine sterile Einwegnadeln verwendet. Dabei wird je nach Symptomatik eine spezifische Kombination aus Akupunkturpunkten benutzt [55], die manuell, durch elektrischen Strom oder Wärme/Hitze stimuliert werden [56].

Insgesamt existieren 20 Meridiane mit mehr als 400 Akupunkturpunkten, wobei heutzutage v. a. zwei Systeme, die 12 Hauptmeridiane (经脉, jing mài) und die 8 außerordentlichen Gefäße (奇经八脉, qí jīng bā mài) angewandt werden [55]. Die 12 Hauptmeridiane teilen sich in zwei Gruppen à 6 Meridiane (阴, yin und 阳, yáng), welche sich in ihrer Flussrichtung (von den Zehen zum Stamm und vom Stamm zu den Fingern) unterscheiden, auf beiden Körperhälften vorkommen, aber nicht kreuzen und sich nochmals auf die jeweiligen Extremitäten (Arme und Beine) à 3 Meridiane untergliedern. Zusammen repräsentieren sie einen Kreislauf, der innerhalb von $24 \mathrm{~h}$ einmal komplett durchlaufen wird. Jeder Meridian erreicht jeweils zu seiner Uhrzeit für $2 \mathrm{~h}$ ein Maximum. Sie sind paarweise einem Sinnesorgan, einer Emotion und einem Gewebe zugeordnet und nach einem Organ benannt (• Abb. 2).

Bezogen auf die Wirksamkeit der Akupunktur werden verschiedene Auffassungen vertreten. Die prospektiv randomisierte Multizenterstudie GERAC („german acupuncture trials“ [57]) verglich zwischen 2002 und 2004 insgesamt 1162 Patienten in drei Armen (echte Akupunktur, Scheinakupunktur, konventionelle Therapie). Dabei zeigte sich nach 6 Monaten eine deutliche Verbesserung der Schmerzsituation durch die Anwendung von Akupunktur im Vergleich zur konventionellen Therapie. Interessanterweise war kein signifikanter Unterschied zwischen der echten Akupunktur und der Scheinakupunktur nachweisbar [57]. Wegen dieser Studie stellt die Akupunktur als Therapieform zur Behandlung von Rü- 


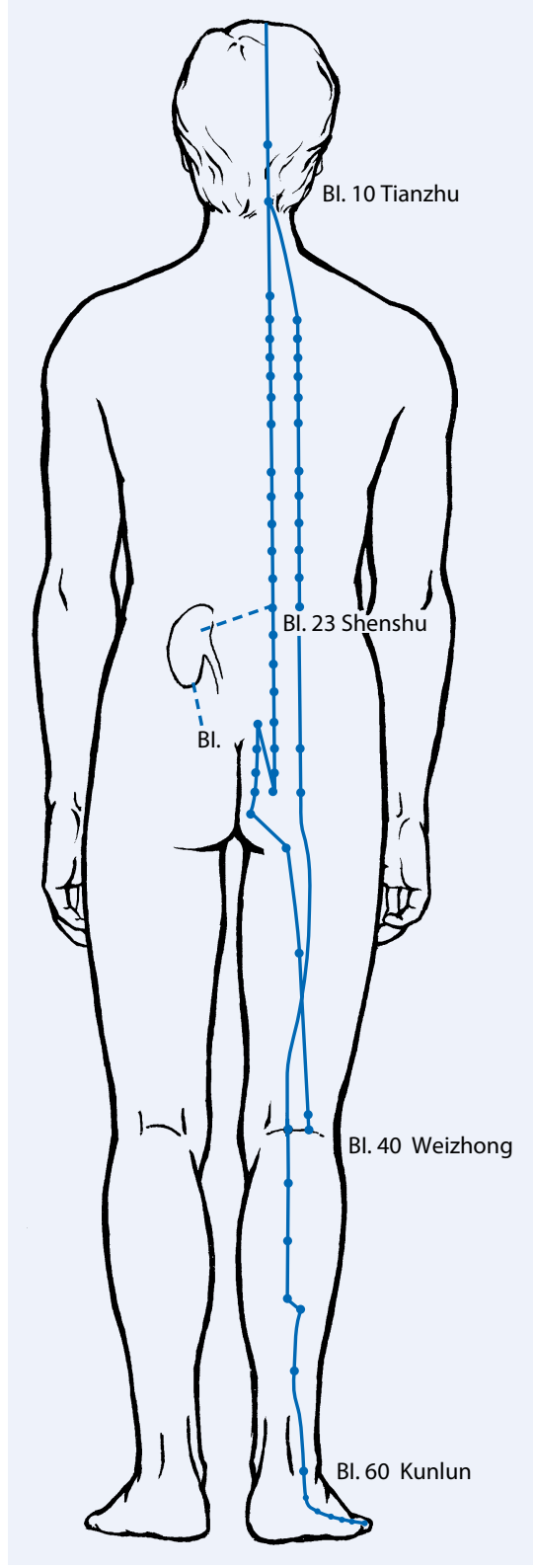

Abb. $2 \Delta$ Harnblasenmeridian. (Aus: Stux et al. [70]: Akupunktur. Springer-Verlag, mit freundl. Genehmigung)

ckenschmerzen und chronischen Kniegelenkschmerzen seit 2007 eine offizielle Kassenleistung in Deutschland dar [58].

Bei der Literatursuche (Stichwörter "acupuncture“ und "chronic pelvic pain syndrome") zeigten sich nach Ausschluss von "case reports" und Veröffentlichungen in einer anderen Sprache als Deutsch oder Englisch nur noch 6 Originalarbeiten $[59,60,61,62,63,64]$ und 5 Übersichtsarbeiten [53, 65, 66, 67, 68]. In den 6 Originalarbeiten, welche zwischen 2003 und 2010 erschienen, wur- den insgesamt 248 männliche CPPS-Patienten mit einem Durchschnittsalter von 38 Jahren eingeschlossen. In nur 2 Studien $[62,63]$ wurde randomisiert (Akupunktur, Scheinakupunktur [63]), wobei eine Studie [62] 3-armig war (Akupunktur, Scheinakupunktur und keine Akupunktur, • Tab. 2).

Methodologisch unterscheiden sich die Akupunkturstudien in Bezug auf die Akupunkturhäufigkeit (ein- [61, 64] oder 2-mal/Woche $[59,60,62,63])$, die Sitzungslänge (10 [61], 20 [60, 62, 64] oder $30 \mathrm{~min} /$ Sitzung $[59,63])$ und die Studiengesamtdauer (4 [61], 6 [59, 60, 62, 64] oder 10 Wochen [63]). Des Weiteren wurde eine unterschiedliche Anzahl von Akupunkturpunkten (1 [61, 64], 3 [62], 4 [59, 63] oder 14 [60]) verwendet.

In allen 6 Studien zeigte sich eine statistisch signifikante (mindestens $\mathrm{p}<0,05$ ) Reduktion der Beschwerdesymptomatik, welche mit Hilfe des standardisierten Fragebogens „National Institute of Health-Chronic Prostatitis Symptom Index" (NIH-CPSI) dokumentiert wurde. Im di- rekten Vergleich Akupunktur vs. Scheinakupunktur [62,63] respektive Akupunktur vs. keine Akupunktur [62] konnte eine signifikante (mindestens $\mathrm{p}<0,05)$ Verbesserung nachgewiesen und die Effektivität der Akupunktur zur Symptomlinderung und Verbesserung der Lebensqualität beim CPPS gezeigt werden.

Keine der 6 Studien berichtete über gravierende Nebenwirkungen während oder nach der Akupunktur, so dass die Akupunktur als sichere Zweitlinientherapie betrachtet werden kann.

In den Übersichtsarbeiten wurden alle oben genannten Originalarbeiten miteinander verglichen. Suh et al. [67] und Westesson et al. [68] bezogen sich auf [62, 63, 64], Lee et al. [65] nahmen zu allen 6 Studien Stellung, Chen et al. [69] kommentierten nur die eigene Arbeit [60], Posadzki et al. [66] verglichen ein Paper [63] mit chinesischen Publikationen und Capodice et al. [53] bezogen sich auf [60, 61].

Die Wirkung der Akupunktur dürfte durch ein Zusammenspiel von verschiedenen Mechanismen an drei Wirkorten

\section{Hier steht eine Anzeige.}

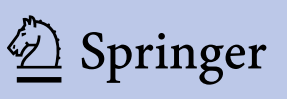


(lokal peripher im Gewebe selbst, spinal und supraspinal) bedingt sein [65].

Zusammenfassend scheint die Akupunktur eine sichere und effektive Behandlungsmöglichkeit darzustellen [65], die als sog. „complimentary and alternative medical" (CAM-)Therapie durchaus beim CPPS angewandt werden kann [53].

\section{Fazit für die Praxis}

- Neurostimulation, Neuromodulation und Akupunktur können bei CPPS-Patienten eine ausgezeichnete Wirkung zeigen und haben ein vorteilhaftes Nebenwirkungsprofil

- Zusätzlich kann durch diese Behandlungsverfahren neben der Schmerzreduktion ggf. auch eine Verbesserung der Sexualfunktion erreicht werden

- Neurostimulation, Neuromodulation und Akupunktur sind unkonventionelle Behandlungsverfahren, die als Zweitlinientherapie bei therapierefraktären CPPS-Patienten eine vielversprechende Therapieoption darstellen und im klinischen Alltag vermehrt eingesetzt werden sollten

\section{Korrespondenzadresse}

\section{PD Dr. T.M. Kessler}

Neuro-Urologie, Zentrum für Paraplegie, Uniklinik Balgrist, Universität Zürich, Forchstraße 340, CH-8008 Zürich, Schweiz tkessler@gmx.ch

Interessenkonflikt. Der korrespondierende Autor gibt fUr sich und seine Koautoren an, dass kein Interessenkonflikt besteht.

\section{Literatur}

1. Engeler D, Baranowski AP, Elneil S et al (2012) EAU guidelines on chronic pelvic pain. http://www.uroweb.org/guidelines

2. Fall $M$, Baranowski $A$, Elneil $S$ et al (2010) EAU guidelines on chronic pelvic pain. Eur Urol 57(1):35-48

3. Krieger J, Nyberg L Jr, Nickel J (1999) NIH consensus definition and classification of prostatitis. JAMA 282(3):236-237

4. NIDDK (2007) Multi-disciplinary approach to the study of chronic pelvic pain (MAPP) research network. http://www.grants.nih.gov

5. International Association for the Study of Pain (2008) Pain - clinical updates, in chronic pelvic and urogenital pain syndromes. Informa Healthcare, New York
6. International Association for the Study of Pain (2003) Pain - clinical updates, in how prevalent is chronic pain? Informa Healthcare, New York

7. The British Pain Society (2006) FAQ - What is the definition of chronic/persistent pain? http://www.britishpainsociety.org/media_faq.htm

8. American Urological Association (2011) Guideline Diagnosis and treatment of IC and BPS. AUA, Linthicum, MD, USA

9. American College of Obstetricians and Gynecologists (2004) Chronic pelvic pain. Obstet Gynecol 103(3):589-605

10. Potts JM, Payne CK (2012) Urologic chronic pelvic pain. PAIN 153(4):755-758

11. Anothaisintawee T, Attia J, Nickel J (2011) Management of chronic prostatitis/chronic pelvic pain syndrome: a systematic review and network meta-analysis. JAMA 305(1):78-86

12. Rodríguez M, Afari N, Buchwald D (2009) Evidence for overlap between urological and nonurological unexplained clinical conditions. J Urol 182(5):21232131

13. McNaughton Collins M et al (2001) Quality of life is impaired in men with chronic prostatitis the Chronic Prostatitis Collaborative Research Network. J Gen Intern Med 16(10):656-662

14. Wenninger K, Heiman JR, Rothman I et al (1996) Sickness impact of chronic nonbacterial prostatitis and its correlates. J Urol 155(3):965-968

15. Sibert L, Rigaud J, Delavierre D et al (2010) Chronic pelvic pain: epidemiology and economic impact. Prog Urol 20(12):872-885

16. Marszalek M, Patard JJ, Carini M et al (2009) Chronic pelvic pain and lower urinary tract symptoms in both sexes: analysis of 2,749 participants of an urban health screening project. Eur Urol 55(2):499508

17. Nickel JC, Downey J, Ardern D et al (2004) Failure of a monotherapy strategy for difficult chronic prostatitis/chronic pelvic pain syndrome. J Urol 172(2):551554

18. Shoskes DA, Katz E (2005) Multimodal therapy for chronic prostatitis/chronic pelvic pain syndrome. Curr Urol Rep 6(4):296-299

19. Fenton BW, Palmieri PA, Boggio P et al (2009) A preliminary study of transcranial direct current stimulation for the treatment of refractory chronic pelvic pain. Brain Stimul 2(2):103-107

20. Stojanovic M (2001) Stimulation methods for neuropathic pain control. Curr Pain Headache Rep 5(2):130-137

21. Herzog J, Weiss PH, Assmus A et al (2006) Subthalamic stimulation modulates cortical control of urinary bladder in Parkinson's disease. Brain 129(12):33663375

22. Herzog J, Weiss PH, Assmus A et al (2008) Improved sensory gating of urinary bladder afferents in Parkinson's disease following subthalamic stimulation. Brain 131(1):132-145

23. Kessler TM, Burkhard FC, Z'Brun S et al (2008) Effect of thalamic deep brain stimulation on lower urinary tract function. Eur Urol 53(3):607-612

24. Hosobuchi Y (1986) Subcortical electrical stimulation for control of intractable pain in humans. J Neurosurg 64(4):543-553

25. Sikiru L, Shmaila H, Muhammed S (2008) Transcutaneous electrical nerve stimulation (TENS) in the symptomatic management of chronic prostatitis/chronic pelvic pain syndrome - a placebo-control randomized trial. Int Braz J Urol 34(6):708-713

26. Kim SW, Paick JS, Ku JH (2007) Percutaneous posterior tibial nerve stimulation in patients with chronic pelvic pain: a preliminary study. Urol Int 78(1):58-62

27. Balken MR van, Vandoninck V, Messelink BJ et al (2003) Percutaneous tibial nerve stimulation as neuromodulative treatment of chronic pelvic pain. Eur Urol 43(2):158-163
28. Pannek J, Nehiba M (2003) Erste Ergebnisse der peripheren Neuromodulation nach Stoller (SANS) bei Blasenfunktionsstörungen. Urologe A 42(11):14701476

29. Balken MR van, Verguns H, Bemelmans BLH (2006) Sexual functioning in patients with lower urinary tract dysfunction improves after percutaneous tibial nerve stimulation. Int J Impot Res 18(5):470-475

30. Wöllner J, Hampel C, Kessler TM (2012) Sacral neuromodulation. BJU Int 110(1):146-159

31. Aboseif S, Tamaddon K, Chalfin S et al (2002) Sacral neuromodulation as an effective treatment for refractory pelvic floor dysfunction. Urology 60(1):52-56

32. Cardarelli S, Flaborea L, Caleffi G et al (2012) Efficacy of sacral neuromodulation on urological diseases: a multicentric research project. Rivista Urol 79(2):9096

33. Comiter CV (2003) Sacral neuromodulation for the symptomatic treatment of refractory interstitial cystitis: a prospective study. J Urol 169(4):1369-1373

34. Elhilali MM, Khaled SM, Kashiwabara T et al (2005) Sacral neuromodulation: long-term experience of one center. Urology 65(6):1114-1117

35. Everaert K, Devulder J, De Muynck M et al (2001) The pain cycle: implications for the diagnosis and treatment of pelvic pain syndromes. Inter Urogynecol J Pelvic Floor Dysfunct 12(1):9-14

36. Everaert K, Kerckhaert W, Caluwaerts H et al (2004) A prospective randomized trial comparing the 1-stage with the 2-stage implantation of a pulse generator in patients with pelvic floor dysfunction selected for sacral nerve stimulation. Eur Urol 45(5):649-654

37. Kessler TM, Buchser E, Meyer S et al (2007) Sacral neuromodulation for refractory lower urinary tract dysfunction: results of a nationwide registry in Switzerland. Eur Urol 51(5):1357-1363

38. Kessler TM, Burkhard FC, Madersbacher $\mathrm{H}$ et al (2008) Safety of prolonged sacral neuromodulation tined lead testing. Curr Med Res Opin 24(2):343-347

39. Maher CF, Carey MP, Dwyer PL, Schluter PL (2001) Percutaneous sacral nerve root neuromodulation for intractable interstitial cystitis. J Urol 165(3):884-886

40. Martellucci J, Naldini G, Carriero A (2012) Sacral nerve modulation in the treatment of chronic pelvic pain. Int J Colorectal Dis 27(7):921-926

41. Otto W, Nowrotek A, Burger M et al (2012) Sacral neuromodulation as second-line treatment strategy for lower urinary tract symptoms of various aetiologies: experience of a German high-volume clinic. Aktuelle Urol 43(3):162-166

42. Peters KM, Feber KM, Bennett RC (2005) Sacral versus pudendal nerve stimulation for voiding dysfunction: a prospective, single-blinded, randomized, crossover trial. Neurourol Urodyn 24(7):643-647

43. Peters KM, Feber KM, Bennett RC (2007) A prospective, single-blind, randomized crossover trial of sacral vs pudendal nerve stimulation for interstitial cystitis. BJU Int 100(4):835-839

44. Peters KM, Konstandt D (2004) Sacral neuromodulation decreases narcotic requirements in refractory interstitial cystitis. BJU Int 93(6):777-779

45. Pham K, Guralnick ML, O'Connor RC (2008) Unilateral versus bilateral stage I neuromodulator lead placement for the treatment of refractory voiding dysfunction. Neurourol Urodyn 27(8):779-781

46. Powell CR, Kreder KJ (2010) Long-term outcomes of urgency-frequency syndrome due to painful bladder syndrome treated with sacral neuromodulation and analysis of failures. J Urol 183(1):173-176

47. Scheepens WA, de Bie RA, Weil EH, van Kerrebroeck PE (2002) Unilateral versus bilateral sacral neuromodulation in patients with chronic voiding dysfunction. J Urol 168(5):2046-2050

48. Siegel S, Paszkiewicz E, Kirkpatrick C et al (2001) Sacral nerve stimulation in patients with chronic intractable pelvic pain. J Urol 166(5):1742-1745 
49. Zabihi N, Mourtzinos A, Maher MG et al (2007) Shortterm results of bilateral S2-S4 sacral neuromodulation for the treatment of refractory interstitial cystitis, painful baldder syndrome, and chronic pelvic pain. Int Urogynecol J Pelvic Floor Dysfunct 19(4):553-557

50. Lavano A, Volpentesta G, Aloisi M et al (2004) Use of chronic sacral nerve stimulation in neurological voiding disorders. J Neurosurg Sci 48(4):157-159

51. Parsons CL (2007) The role of the urinary epithelium in the pathogenesis of interstitial cystitis/prostatitis/ urethritis. Urology 69(Suppl 4):9-16

52. Peters KM, Killinger KA, Boguslawski BM et al (2010) Chronic pudendal neuromodulation: expanding available treatment options for refractory urologic symptoms. Neurourol Urodyn 29(7):1267-1271

53. Capodice JL, Bemis DL, Buttyan R et al (2005) Complementary and alternative medicine for chronic prostatitis/chronic pelvic pain syndrome. Evid Based Complement Alternat Med 2(4):495-501

54. NIN Consensus Development Panel on Acupuncture (1998) Acupuncture. JAMA 280(17):1518-1524

55. Deadman P, Baker K, Al-Khafaji M (1998) A manual of acupuncture. J Chin Med, ISBN-10: 0951054678

56. Ellis A, Wiseman N, Boss K (1991) Fundamentals of Chinese acupuncture. Paradigm Pubns, Brookline, Massachusetts, USA

57. Haake M, Müller HH, Schade-Brittinger C et al (2007) German Acupuncture Trials (GERAC) for chronic low back pain: randomized, multicenter, blinded, parallel-group trial with 3 groups. Arch Intern Med 167(17):1892-1898

58. Bundesministerium für Gesundheit (2006) Bekanntmachung eines Beschlusses des Gemeinsamen Bundesausschusses über eine Änderung der Richtlinie Methoden vertragsärztliche Versorgung in Anlage I Anerkannte Untersuchungs- oder Behandlungsmethoden" und in Anlage II „Methoden, die nicht als vertragsärztliche Leistungen zu Lasten der Krankenkassen erbracht werden dürfen". Akupunktur

59. Capodice JL et al (2007) A pilot study on acupuncture for lower urinary tract symptoms related to chronic prostatitis - chronic pelvic pain. Chin Med 2(1):1

60. Chen R, Nickel JC (2003) Acupuncture ameliorates symptoms in men with chronic prostatitis/chronic pelvic pain syndrome. Urology 61(6):1156-1159

61. Honjo H et al (2004) Effects of acupuncture for chronic pelvic pain syndrome with intrapelvic venous congestion - preliminary results. Int J Urol 11(8):607-612

62. Lee SH, Lee BC (2009) Electroacupuncture relieves pain in men with chronic prostatitis/chronic pelvic pain syndrome: three-arm randomized trial. Urology 73(5):1036-1041

63. Lee SWH et al (2008) Acupuncture versus sham acupuncture for chronic prostatitis/chronic pelvic pain. Am J Med 121(1):79.e1-79.e7

64. Tugcu $V$ et al (2010) Effectiveness of acupuncture in patients with category IIIB chronic pelvic pain syndrome-a report of 97 patients. Pain Med 11(4):518-523

65. Lee SH, Lee BC (2011) Use of acupuncture as a treatment method for chronic prostatitis/chronic pelvic pain syndromes. Curr Urol Rep 12(4):288-296

66. Posadzki P et al (2011) Acupuncture for chronic nonbacterial prostatitis/chronic pelvic pain syndrome: a systematic review. J Androl 33(1):15-21

67. Suh LK, Lowe FC (2011) Alternative therapies for the treatment of chronic prostatitis. Curr Urol Rep 12(4):284-287

68. Westesson KE, Shoskes DA (2010) Chronic prostatitis/chronic pelvic pain syndrome and pelvic floor spasm: can we diagnose and treat? Curr Urol Rep 11(4):261-264

69. Chen R, Nickel JC (2004) Acupuncture for chronic prostatitis - chronic pelvic pain syndrome. Curr Urol Rep 5:305-308

70. Stux G (2007) Akupunktur. Springer, Berlin Heidelberg New York

\title{
Galenus-von- Pergamon-Preis 2012
}

\author{
Die Jury hat entschieden
}

Für manche ist er der inoffizielle „Nobelpreis" für Pharmakologie: der Galenus-von-Pergamon-Preis. Am 18. Oktober wurde er im Rahmen einer festlichen Gala erneut verliehen - an drei beeindruckende Preisträger.

Primary Care

Der Preis in der Kategorie Primary Care würdigt ein Medikament, das bei einer breiten Patientengruppe eingesetzt wird. In diesem Jahr hat Novartis Pharma diesen Preis für Gilenya ${ }^{\circledR}$ (Fingolimod) erhalten. Fingolimod ist ein orales Medikament zur Therapie von Patienten mit Multipler Sklerose (MS). Es ist zugelassen für bisher nicht behandelte Patienten, die an einer rasch fortschreitenden, schweren schubförmigen MS erkrankt sind. Es ist zudem indiziert zur Eskalationstherapie, wenn trotz Behandlung mit einem Beta-Interferon eine hohe Krankheitsaktivität vorliegt. Mit Fingolimod gelingt es, die Schubrate zu verringern.

\section{Specialist Care}

Der Preis in der Kategorie Specialist Care zeichnet ein Medikament aus, das zur Behandlung seltener Erkrankungen verwendet wird. Der diesjährige Gewinner ist Zelboraf ${ }^{\oplus}$ (Vemurafenib) von Roche Pharma. Vemurafenib ist die erste Option für eine personalisierte Therapie bei inoperablem oder metastasiertem Melanom. Das Medikament in Form von Filmtabletten ist zugelassen zur Therapie von Melanompatienten, die ein mutiertes BRAF-Gen haben. In der Zulassungsstudie betrug die geschätzte mediane progressionsfreie Überlebenszeit 5,6 Monate im Vergleich zu Patienten mit der Standardchemothera- pie mit Dacarbazin. In der VemurafenibGruppe war zudem das Sterberisiko um $63 \%$ und das Progressionsrisiko um 74\% verringert.

\section{Grundlagenforschung}

In der Kategorie Grundlagenforschung wurde das Team um Dr. Thomas Worzfeld aus Bad Nauheim für die Entwicklung eines neuen Ansatzes zur Therapie bei metastasierendem Brustkrebs geehrt. Worzfeld und sein Team haben herausgefunden, dass der Rezeptor Plexin-B1 eine besondere Bedeutung für die Metastasierung bei Brustkrebs hat. Anhand von Gewebeproben von Patientinnen mit einem Mammakarzinom stellten sie fest, dass die Frauen eine umso bessere Überlebenschance hatten, je weniger Rezeptoren im Tumorgewebe vorhanden waren. Inzwischen steht ein monoklonaler Antikörper gegen diesen Rezeptor zur Verfügung, der derzeit präklinisch getestet wird.

\section{Springer Medizin CharityAward 2012}

Das Kinder- und Jugendhospiz Balthasar in Olpe ist für sein unermüdliches Engagement mit dem Springer Medizin CharityAward ausgezeichnet worden. Balthasar wurde im Jahr 1998 gegründet und ist damit das erste Kinder-und Jugendhospiz in Deutschland. Der von Schirmherr Daniel Bahr überreichte Springer Medizin CharityAward umfasst einen Barscheck über 50.000 Euro und ein Medienpaket über weitere 100.000 Euro. Damit unterstützt die Fachverlagsgruppe den Gewinner gezielt bei seiner Öffentlichkeitsarbeit.

Quelle: Springer Medizin 